\title{
Retrieval processes in perceptual recognition and cued recall: The influence of category size
}

\author{
DOUGLAS L. NELSON, CATHY L. McEVOY, and MARIA TERESA BAJO \\ University of South Florida, Tampa, Florida
}

\begin{abstract}
The purpose of these experiments was to determine whether nonsemantic category size and other variables would influence perceptual recognition as they influence cued recall. Procedures developed by Jacoby and his associates were replicated with words belonging to either small or large nonsemantic categories and with recognition tested under bright-target/patterned-mask conditions. The results indicated that words belonging to larger nonsemantic sets were more difficult to identify. This finding and the results of other manipulations are discussed in relation to the interactive activation model and to the proposal that perceptual recognition performance is dependent on a retrieval process similar to that involved in recall prompted by nonsemantic cues.
\end{abstract}

Studying or merely reading a word facilitates its perceptual identification even after a period of several days (Jacoby, 1983; Jacoby \& Dallas, 1981; Jacoby \& Witherspoon, 1982). Jacoby and his associates explain this effect with the assumption that nonsemantic information gained from the identification task combines with nonsemantic information preserved from prior episodic study. This process enables subjects to retrieve more information from studied than from new words. Because this perceptual enhancement is sensitive to manipulations of retrieval conditions, Jacoby (1983) concludes that both perceptual and memory tasks rely on the same memory representation system.

One implication of this view is that perceptual recognition and recall cued by nonsemantic cues might share a common retrieval process. Variables that influence the effectiveness of nonsemantic cues in recall tasks should tend to have similar effects in perceptual recognition. For example, two potentially important variables are the category size defined by the retrieval cue and the strength of the relationship between the cue and its target. In cuedrecall tasks, a cue that identifies a target within a relatively large set of related items is not nearly as effective as one that identifies it as a member of a smaller set (e.g., Nelson \& McEvoy, 1979a, 1979b; Nelson, McEvoy, \& Friedrich, 1982). Hence, the greater the number of lexical alternatives defined by the cue, the worse the recall. Furthermore, the weaker the connection between the cue and its target, the worse the recall (e.g., Nelson \& McEvoy, 1979a, 1979b).

This research was supported by Grant MH 16360 to the first author from the National Institute of Mental Health. The authors are indebted to Larry Jacoby and an unnamed reviewer for their comments and to Jim Simpson and Marion Robinson for collecting the data. Requests for reprints should be sent to Douglas Nelson, Department of Psychology, University of South Florida, Tampa, FL 33620.
In these experiments, relative category size and strength are estimated from normative data. For example, with nonsemantic categories, words or word endings are presented to large groups of students who are asked to write the first word that comes to mind that rhymes with the presented cue (Nelson \& McEvoy, 1979b). Category size is then estimated by counting the number of different rhyming words, and the relative strength of any given word is estimated by calculating the proportion of subjects giving it. For instance, the cue ES defines a relatively large set of 20 items that includes MESS, LESS, DRESS, etc., and the cue ǓSK defines a smaller set of 5 items that includes TUSK, MUSK, DUSK, etc. Of the 184 subjects in the sample, $27 \%$ gave MESS, $16 \%$ gave LESS, etc.

In the next phase, other subjects are given a single study trial on a list of words, with each item having been taken from a different rhyme category. This study trial is followed by a test trial in which the endings of the studied words are shown to aid recall. If the study list contained the words MESS and TUSK, their respective test cues would be ESS and USK. As mentioned previously, the results obtained with such cues indicate that endings defining larger sets are less effective, as are endings that are more weakly related to their targets. These effects are found regardless of whether or not nonsemantic features were emphasized during study.

The specific purpose of the present experiments was to determine whether similar effects would be apparent in perceptual identification. To accomplish this aim, the study procedures used in conjunction with the cued-recall experiments were replicated. The to-be-remembered target words belonged to either large or small normatively defined nonsemantic sets, and the endings of these words were either strongly or weakly related to their targets. In Experiment 1, these targets were shown either in the presence of a context that emphasized nonsemantic fea- 
tures or in the absence of such context (e.g., CHESS MESS vs. MESS alone). Each target was then briefly exposed under bright-target/patterned-mask conditions, and subjects were asked to identify the item presented. Finally, to complete the parallel with the procedures developed by Jacoby and Dallas (1981), printed word frequency and prior task experience were factorially manipulated with all variables.

We expected to find that prior study would facilitate identification and that this effect would reduce the typical advantage found for high-frequency words in this task (Jacoby \& Dallas, 1981). Furthermore, we expected to find category size effects to the extent that perceptual identification relies on a retrieval process similar to that associated with recall cued by nonsemantic cues. That is, these effects were expected regardless of whether or not nonsemantic information was emphasized at study. They would also be expected for new words on the assumption that the brief exposure of a new word produces a trace that is disrupted by the mask. Information that survives the mask is then used to provide constraints on the pool of potential candidates. The larger the set, the less the chances for successful recall. Hence, this retrieval view suggests that category size effects should be equally apparent under all conditions.

Somewhat different expectations for the category size outcome can be derived from the interactive activation model developed by McClelland and Rumelhart (1981). The basic assumption of this conceptualization is that knowledge about a word interacts with incoming featural information to codetermine the nature and time course of its perception. The presentation of a word on the perceptual identification task initiates the process of activating detectors for letters consistent with input at the featural level. As these activations increase in strengh, they start activating detectors for words that are consistent with letters, and, in turn, word activation feeds back to the letter level to reinforce the identification of letters within the word. This interactive model would presumably explain the study effect as resulting from "top-down" processing. Prior study of the word strengthens it at the word level, and this recent activation conceivably could produce feedback at the letter level to promote identification on the later perceptual test.

This model predicts that minimal set size effects will be evident and that these effects will be reduced by prior study of the target. Briefly, when a new word is shown on the identification test, it produces a weak activation of other words that share letters with it. On the reasonable assumption that more competing words are activated for targets having larger sets, identification for these items should differentially suffer. McClelland and Rumelhart (1981) referred to these competing items as "enemies." One reason for predicting minimal effects of set size is that the nodes for the enemies receive inhibitory inputs from beginning letters that are not shared with the target. This inhibition weakens their influence. Moreover, these minimal set size effects should be reduced further if the targets have recently been activated during a prior study phase. Prior study should strengthen targets at the word level, and this strengthening should reduce, if not eliminate, the influence of enemies. In contrast, the influence of these enemies should be made more evident if nonsemantic features are emphasized during the study trial (e.g., CHESS MESS vs. MESS alone). Such an emphasis should especially strengthen the endings of the targets. Hence, this interaction model suggests that set size effects will interact with prior study and with study context. Minimal effects should be observed with new words, they should be eliminated when the target is studied alone, and they should be enhanced when nonsemantic information is emphasized at study.

Finally, it should be noted that the relative strength of the relationship between the endings and their targets was also varied. Endings were either very strongly related or only weakly related to their respective targets. The primary reason for including this manipulation as to complete the parallel with our earlier work in cued recall (e.g., Nelson \& McEvoy, 1979a, 1979b). As in that task, we expected stronger endings to facilitate identification. However, this facilitation was expected to be substantially reduced in the perceptual task because of the presence of initial letters in the cue. This reduction could be predicted from the retrieval conceptualization, since information contributed from beginning letters should provide critical input. This issue, along with other questions, was explored further in Experiments 2 and 3. Experiment 2 involved a more direct comparison of cued recall and perceptual identification. Strength, set size, and word frequency were varied, but only the endings of the words were presented as cues. They were available for as long as the subject wished or for 400 or $150 \mathrm{msec}$ under brighttarget/patterned-mask conditions. Experiment 3 involved a direct comparison between words and endings as stimuli, with the same word characteristics being manipulated.

\section{EXPERIMENT 1}

\footnotetext{
Method

Design. The experimental design conformed to a $2^{5}$ mixed-model factorial. Presence-absence of rhyme context cues during study was manipulated as a between-subjects variable, and prior study (old vs. new), printed word frequency, category size, and rhyme strength were all manipulated as within-subject variables.

Materials. Rhyme norms were collected for 396 word endings. Each ending was presented via a tape recorder to large groups of subjects $(\mathrm{n}=153-242)$. Each subject was instructed to write the first word that came to mind that rhymed with the sound just presented. Their responses were then used to estimate category size and rhyme strength. Size was estimated by counting the total number of different but appropriate rhyming words, and relative strength was estimated by calculating the probability that any given response was provided (see Nelson \& McEvoy, $1979 \mathrm{~b}$, for additional details).

These norms were used to construct two different study lists of 48 target words. The words of each list could be divided into eight subsets representing all combinations of word frequency, set size, and relative strength in relation to the word ending. Each subset contained 6 words. With this procedure, there were 24 items per list representing each manipulated within-subject variable. Using the L-count (Thorndike \&
} 
Lorge, 1944), word frequency per 4.5 million words averaged 45.79 $(S D=34.87)$ and $1,191.92(S D=1,378.71)$, respectively, for lowand high-frequency words. Three of the high-frequency words were rated as $\mathrm{A}$, and the remaining 45 words in the two lists were rated as $\mathrm{AA}$ in the G-count. Rhyme set size averaged $5.81(\mathrm{SD}=1.57$ ) and 19.63 ( $S D=4.70$ ), for small and large set sizes. A separate count of only those rhyme items that overlap visually (e.g., HUSK TUSK) yielded similar estimates of $4.06(\mathrm{SD}=2.11)$ and $12.04(\mathrm{SD}=6.06)$ for small and large sets. Relative strength averaged $.03(\mathrm{SD}=.02)$ for weak and $.30(\mathrm{SD}=.09)$ for strong targets. With few exceptions, strong rhymes were given as the most likely responses to their normative cues. Finally, each of these variables was carefully equated at both levels of the other variables. In addition, word length was equated as closely as possible in all conditions. Overall, it averaged $4.76(\mathrm{SD}=.65)$ letters. Individual means for low and high frequency, small and large sets, and strong and weak items were, respectively, 4.71 and $4.81,4.94$ and 4.58 , and 4.60 and 4.92 .

Procedure. All subjects received a single study-test trial. During study, the target words were presented in a tachistoscope at a 3-sec rate that was paced by an auxiliary timer. At each click of the timer, the experimenter changed the stimulus, and this procedure reduced an effective exposure of approximately $1.5 \mathrm{sec}$. All items were typed on white index cards with an IBM Orator element and were attached to specially constructed Masonite holders. Each stimulus was centered at fixation and spanned approximately $1.5^{\circ}$ of visual arc in the cue-absent condition and approximately $3.0^{\circ}$ when targets appeared juxtaposed with nonsemantic context cues.

When context cues were present during study, they were underlined and appeared to the left. The subjects were told to read all items aloud from left to right, to remember as many targets as possible, and to concentrate on the rhyme relationship because the cues would be presented as memory aids later on. Of course, to bias the encoding of their respective targets, these rhyme context cues were presented only during study. They never appeared on the perceptual recognition test. When contextual cues were absent at study, the subjects were simply told to read each item aloud and to remember as many items as possible. No information about testing was provided. The study sequence was blocked so that every eight items presented one item from each condition. This ordering was unsystematically randomized for each subject.

During the test phase, studied words and new words were intermixed and were shown for $40 \mathrm{msec}$ each in the perceptual recognition task. Studied (old) words consisted of targets shown during the study trial, either List 1 or List 2. New words consisted of targets from the list not shown during study. Hence, if List 1 was studied, targets from List 2 served as new words and vice versa. With this procedure, the old-new manipulation crossed all other variables. The test items were blocked into groups of 16 so that each condition was represented once in each block. This ordering was different from the study sequence, and it was changed for each subject.

The subjects were informed that words would be flashed in front of them and that they were to identify each word immediately after its presentation. They were told that they had just seen some of the words in the previous task. Guessing was strongly encouraged. The subjects were told that they would be controlling when each word was exposed. In order to do this, the instructions asked them to focus on a fixation cross whenever they heard the ready signal. As soon as they felt ready, they could press a button that would briefly flash the word. The word was presented $350 \mathrm{msec}$ after the button was pushed. They were also told that a masking stimulus would be presented immediately after the target. The mask consisted of three rows of superimposed Xs and Os, and it remained on the screen for $2 \mathrm{sec}$ before being replaced by the cross, which was displayed on a lighted field. This sequence was repeated until the entire list was shown.

Before the experimental test list was presented, the subjects were given a practice list of 10 words to train them in the task. All of these words were new, and the first item was shown for $100 \mathrm{msec}$ so that nearly all the subjects could identify it. The durations for Items 2-6 were each reduced by $10 \mathrm{msec}$ so that Items 7-10 were flashed at $40 \mathrm{msec}$, the duration used for the experimental list. As soon as the practice task was completed, the subjects started working on the main list.
Subjects. Thirty-two subjects participated in the experiment, with 16 assigned to each cue-presence condition. These subjects were selected from courses in introductory psychology under an incentive system, and they were randomly assigned to one of the two conditions.

\section{Results}

Table 1 shows the probabilities of correct perceptual identification as a function of the principal conditions of the experiment. The first notable characteristic of these values is that they replicate findings reported by Jacoby and Dallas (1981). Despite the use of a different display format and other minor procedural differences, the results are very similar. First, studying words just prior to the identification task facilitated their recognition. The respective probabilities of identifying old and new words were .47 and .36 , and this difference was reliable $[F(1,30)=$ $46.18, \mathrm{MSe}=.036]$. Second, high-frequency words (.46) were more likely to be correctly identified than lowfrequency words $(.37)$. This difference was also reliable $[F(1,30)=21.75$, MSe $=.049]$. Finally, the effects of study presentation and frequency interacted as expected $[F(1,30)=11.81, \mathrm{MSe}=.037]$. This interaction indicated that the effect of printed word frequency was substantially reduced for studied words. The probabilities of correct identification were .43 and .28 for high- and lowfrequency new words and .49 and .45 for high- and lowfrequency old words. Thus, with a single study presentation, low-frequency words were nearly as likely to be recognized as high-frequency words.

For present purposes, the more important findings concern potential effects of category size. As can be seen in Table 1, target words belonging to larger nonsemantic sets were less likely to be identified than those belonging to smaller sets. The probability of identifying targets having larger sets was .37 , and the probability of recognizing targets having smaller sets was .46. This difference was significant $[\mathrm{F}(1,30)=28.36, \mathrm{MSe}=.033]$. This effect of category size was independent of whether or not the words were studied $(F<1)$. Furthermore, it was independent of whether or not rhyme information was emphasized during study $(\mathrm{F}<1)$.

The results of the statistical analysis of these data also indicated that neither the presence of contextual cues during study $(F<1)$ nor the relative strength between the

Table 1

Probability of Correct Identification as a Function of Strength, Frequency, Category Size, Prior Study, and the Presence of Rhyme Cues During Study

\begin{tabular}{|c|c|c|c|c|c|c|c|c|c|}
\hline \multirow[b]{3}{*}{ Rhyme } & \multirow{3}{*}{$\begin{array}{l}\text { Prior } \\
\text { Study }\end{array}$} & \multicolumn{4}{|c|}{ Strong } & \multicolumn{4}{|c|}{ Weak } \\
\hline & & \multicolumn{2}{|c|}{ High } & \multicolumn{2}{|c|}{ Low } & \multicolumn{2}{|c|}{ High } & \multicolumn{2}{|c|}{ Low } \\
\hline & & Small & Large & Small & Large & Small & Large & Small & Large \\
\hline Absent & $\begin{array}{l}\text { Old } \\
\text { New }\end{array}$ & & & & & $\begin{array}{l}.55 \\
.48\end{array}$ & $\begin{array}{l}.48 \\
.34\end{array}$ & & $\begin{array}{l}.50 \\
.30\end{array}$ \\
\hline Present & $\begin{array}{l}\text { Old } \\
\text { New }\end{array}$ & $\begin{array}{l}.46 \\
.49\end{array}$ & $\begin{array}{l}.48 \\
.44\end{array}$ & $\begin{array}{l}.50 \\
.31\end{array}$ & $\begin{array}{l}.29 \\
.21\end{array}$ & $\begin{array}{l}.48 \\
.43\end{array}$ & $\begin{array}{l}.41 \\
.32\end{array}$ & $\begin{array}{l}.50 \\
.27\end{array}$ & $\begin{array}{l}.40 \\
.27\end{array}$ \\
\hline & Mean & .49 & .47 & .43 & .26 & .49 & .39 & .42 & .37 \\
\hline
\end{tabular}


endings and their targets ( $F<1)$ had any effect on performance. Of the remaining 26 sources of variance, only 2 were significant. These were the interactions of frequency $\times$ strength $[\mathrm{F}(1,30)=11.64, \mathrm{MSe}=.024]$ and category size $\times$ frequency $\times$ strength $[F(1,30)=9.54$, $\mathrm{MSe}=.031]$. These interactions appeared to be related to especially poor recognition performance for items falling in the strong, low-frequency, large set size condition. The three-way interaction is illustrated in the bottom row of Table 1. The reasons for this poor performance are unclear, and since the effect was not replicated in Experiment 3 with the same materials, it will not be considered further.

\section{Discussion}

These results indicate that studied words are more likely to be identified under perceptually difficult conditions than are new words. Moreover, a single study presentation was sufficient to nearly eliminate the word-frequency advantage usually found in this task. These findings replicate those reported by Jacoby and Dallas (1981) and extend them to a different presentation format and new materials. As they suggest, studying words allows subjects to retrieve more nonsemantic information under perceptually difficult conditions, and this seems to be particularly true for low-frequency words. Given the interactive feedback assumption of the McClelland and Rumelhart (1981) model, these results also seem to be quite compatible with this formulation.

Perhaps the most important finding in Experiment 1 is the consistency with which category size effects are obtained. Although effects are not very large, targets having larger sets of enemies are consistently more difficult to recognize unnder bright-target/patterned-mask conditions. These results stand in apparent contrast to the frequently cited findings reported by Johnston (1978). He found that, under similar conditions, weakly constrained words (larger sets) were just as likely to be identified as highly constrained words (smaller sets). However, there is a major difference in the manner in which lexical constraint is measured in the two studies. Johnson restricted his count to include only four-letter words sharing particular letters. The problem with this procedure is that it assumes that the memory system can restrict activation to a word population having a specified length. To the extent that activation of related items is automatic, this assumption seems dubious. Subjects may have confined their responses to four-letter words, but there is no reason to believe that this means that the system confines activation to words of this length. Recomputation of the set sizes for his "Letter Position 1" stimuli with our normative data suggests that the range of the constraint manipulaton in his experiment may have been too narrow. If the count is restricted to all words in the category sharing the same ending regardless of word length, then the large categories average $13.26(\mathrm{SD}=4.32)$ and the small categories average $8.03(\mathrm{SD}=6.15)$. Given the overlap between the distributions, this difference seems quite small. The point is that Johnston's (1978) inference that contextual constraint or set size has no effect on freechoice identification may be invalid. Others have reached a similar conclusion (e.g., Paap, Newsome, McDonald, \& Schveneveldt, 1982).

The set size effects observed in Experiment 1 did not depend on whether or not words were studied in the context of cues that emphasized nonsemantic features. And they did not depend on whether the items were studied at all. Category size effects were equivalent for studied and new words. Unlike the effects of word frequency, the effects of category size were not reduced by prior study. The Mr 'alland and Rumelhart (1981) model suggests that wor, belonging to larger sets should have differentially benefited from a recent study experience. The influence of the enemies of the target words should have been differentially reduced for these items.

These findings indicate that category size has parallel effects in rhyme-cued recall and perceptual identification. They suggest that perceptual identification may be dependent on a retrieval process similar to that associated with recall cued by nonsemantic cues. As Jacoby (1983) argued, both perceptual and memory tasks may be dependent on the same memory representation system. However, despite this parallel, Experiment 1 also reveals an important contrast in findings. In cued-recall tasks, relative preexperimental strength has substantial effects on recall (e.g., Nelson \& McEvoy, 1979a, 1979b), but in perceptual recognition, it seems to have little or no influence. The source of this difference was initially unclear, but we thought it might be related to the nature of the "cue" presented in the two tasks. In cued recall, only the ending of the target is provided, and in perceptual recognition, the target itself serves as the cue. In the identification task, it is possible for subjects to retrieve some or even complete information on beginning letters. Such information may have served to reduce the influence of strength.

\section{EXPERIMENT 2}

The primary purpose of Experiment 2 was to test the hypothesis that the absence of strength effects in perceptual recognition was related to the availability of information associated with beginning letters. As in Experiment 1, the target words presented in the study list varied in category size, strength, and word frequency. At test, only the endings of these targets were provided as cues, and they were exposed for one of three intervals. They were available until the subjects had produced a response, or they were presented for 400 or for $150 \mathrm{msec}$ and were followed by a patterned mask. Exposure duration was varied in this way to provide a direct comparison between cued recall and perceptual recognition. The self-paced exposure was intended to replicate previously used cued-recall conditions. The $400-\mathrm{msec}$ condition with the mask was meant to produce an overall level of performance that would be similar to that found 
with cued recall, but under conditions more closely approximating perceptual recognition. Finally, the $150-\mathrm{msec}$ condition with the mask was intended to provide the subjects with a very difficult identification task. This rate was selected on the basis of pilot research to produce a level of performance that was generally below that found in Experiment 1 for whole-word stimuli. The purpose of selecting such a rate was to ensure that strength effects, if obtained, could not be attributed simply to higher levels of overall identification.

Expectations regarding the effects of strength hinged on considerations of task versus initial letter. On the one hand, if strength effects are task dependent, then such effects should be obtained under the self-paced procedure and maybe at the $400-\mathrm{msec}$ exposure, but not at the 150 -msec exposure. On the other hand, if strength effects are eliminated in identification only because of the presence of initial letters, then strength effects should be apparent at all exposure durations. Ending cues provide no information in initial letter positions. Strength effects may be reduced at the shorter duration because of floor effects, but they should still be apparent if the initial letter hypothesis is correct.

\section{Method}

Design. The experimental design formed a $3 \times 2 \times 2 \times 2$ mixedmodel factorial. The three levels of exposure time were manipulated between subjects, and the within-subject variables included category size, relative ending-to-target strength, and printed word frequency.

Materials and Procedure. The lists of target words and the procedures used for the study trial were identical to those used in Experiment 1. However, it might be noted that two variables manipulated in that study were omitted in this one. The condition with rhyme context cues present at study was dropped so that targets always appeared alone. In addition, endings for new words were never presented during the test trial in this experiment.

Test-trial procedures used for the self-paced exposure condition were similar to those developed for cued recall (e.g., Nelson \& McEvoy, 1979b). Each ending was exposed in the scope, and the subjects read it aloud before attempting to recall its target. However, all subjects in this condition were required to work on a series of multiplication problems before beginning the recall test. The purpose of this task was to approximately equate test delay in all conditions. The training period in the perceptual identification task required about $5 \mathrm{~min}$.

Test-trial procedures used in the 400- and 150-msec conditions were similar to those used for Experiment 1. However, the subjects received training on 10 endings instead of 10 words. They were told to pronounce aloud the ending seen. In the experimental task, they were told to pronounce the ending and then to try to identify the word from the study list from which the ending came. In this experiment, blanks were not used in place of the missing initial letters. No feedback was provided on the accuracy of recall in any condition.

Subjects. Forty-eight subjects participated, with 16 subjects being assigned to each condition of exposure duration. Eight of these students were assigned to List 1 , and the remaining 8 were assigned to List 2 . All subjects were selected from the same source and assigned to conditions as in Experiment 1.

\section{Results}

Probabilities of correct identification for the principal conditions are shown in Table 2. Examination of these values will show that the 150 -msec exposure substantially reduced the chances of identifying the target and that both category size and strength effects are evident in every con-
Table 2

Probability of Correct Identification as a Function of Strength, Frequency, Category Size, and Exposure Duration

\begin{tabular}{|c|c|c|c|c|c|c|c|c|c|}
\hline \multirow[b]{3}{*}{ Exposure } & \multicolumn{4}{|c|}{ Strong } & \multicolumn{4}{|c|}{ Weak } & \\
\hline & \multicolumn{2}{|c|}{ High } & \multicolumn{2}{|c|}{ Low } & \multicolumn{2}{|c|}{ High } & \multicolumn{2}{|c|}{ Low } & \\
\hline & Small & Large & Small & 1 Large & Small & Large & Small & Large & Mean \\
\hline Self-Paced & .91 & .61 & .81 & .77 & .58 & .36 & .58 & .38 & .63 \\
\hline & .91 & .68 & .78 & .66 & .56 & .26 & .52 & .23 & .58 \\
\hline $150 \mathrm{msec}$ & .35 & .30 & .34 & .26 & .17 & .08 & .24 & .14 & .24 \\
\hline
\end{tabular}

dition. The analysis of variance indicated that duration $[\mathrm{F}(2,45)=62.01, \mathrm{MSe}=.093]$, category size $[\mathrm{F}(1,45)$ $=105.92, \mathrm{MSe}=.026]$, and strength $[\mathrm{F}(1,45)=253.33$, $\mathrm{MSe}=.028]$ were all reliable sources of variance. Fisher's two-tailed least significant difference (LSD) for duration was .08 and indicated that the 150 -msec duration differed from the 400 -msec and self-paced conditions, which did not differ from each other.

This analysis also indicated that three interactions were reliable. Duration interacted with both category size $[F(2,45)=8.29]$ and strength $[F(2,45)=12.64]$. Fisher's LSD for each of these effects was .06. These interactions reflect the fact that both set size and strength effects were weaker at the 150 -msec duration than at the other durations. However, it is important to note that both variables still had reliable effects at this duration. The interactions appeared to be reflecting floor effects rather than the absence of effects. Mean correct target identifications in the self-paced, $400-\mathrm{msec}$, and 150 -msec conditions for targets having small and large sets were, respectively, .72 and $.53, .69$ and .46 , and .28 and .20 . For strong and weak ending-to-target relationships, these respective means were .78 and $.48, .76$ and .39 , and .31 and .16 .

The analysis also indicated that the category size $x$ strength interaction was reliable $[F(1,45)=4.29$, MSe $=.024$, Fisher's LSD $=.05]$. This interaction indicated that the influence of category size was somewhat reduced when endings were more strongly related to their targets. Mean correct identifications for small and large sets were .68 and .44 when endings were strongly related and .55 and .24 when they were weakly related. This pattern has been observed previously when word endings were used in cued recall (Nelson et al., 1982), but it has not been invariably found (Nelson \& McEvoy, 1979b). Presumably, strong-ending cues can reduce the extent of search for target information, thus reducing the category size effect (Nelson, 1981).

Finally, none of the remaining sources, including frequency, even approached the criterion for significance. The absence of frequency effects was to be expected, since all targets were old. This null result replicates Experiment 1 and the data reported by Jacoby and Dallas (1981).

\section{Discussion}

The results of Experiment 2 indicate that the category size defined by an ending cue and its strength in relation to the target can be important determinants of both cued 
recall and perceptual identification under brighttarget/patterned-mask conditions. The absence of strength effects in Experiment 1 cannot readily be attributed to differences in task. Instead, this absence appears to be related to the presence of initial letters. Strength effects are not obtained when the target itself is exposed, and they are obtained when only its ending is shown. One implication of this finding is that information gained from initial letters serves to change the nature of the search for encoded target information in the perceptual identification task. This interpretation suggests that the same retrieval processes are involved in perceptual identification that are involved in recall cued with nonsemantic cues. The magnitude of the effects of variables manipulated in the two tasks may differ, but this difference may be related more to differences in cue information than to basic differences in processes associated with each task. This point was explored further in Experiment 3.

\section{EXPERIMENT 3}

Experiment 3 provided a direct comparison between words and endings in the perceptual identification task. As in Experiment 1, stimuli varied in category size, strength, frequency, and whether they were old or new. Half of the subjects were shown words on the identification test, and half were shown the endings of these words. Although it would have been ideal to present each stimulus type at the same exposure duration, this was impossible because of the word superiority effect (e.g., Reicher, 1969). Endings presented at $40 \mathrm{msec}$ under brighttarget/patterned-mask conditions appear as unrecognizable blurs. Because of this problem, stimulus type and duration were intentionally confounded. Words were shown for $40 \mathrm{msec}$ and endings for $250 \mathrm{msec}$ prior to the onset of the mask. The exposure duration selected for endings had been empirically determined in pilot work undertaken to obtain equal recognition performance for words and their endings on new words. The elimination of overall differences for these items was expected to provide a fair basis for assessing the relative magnitudes of set size and strength effects for the two kinds of stimuli.

We expected this experiment to replicate the study effect, the word frequency effect, and their interaction. We also expected to find that category size and strength effects would be present but reduced when words were used instead of endings. Such reductions could be explained by either the perceptually based or retrieval viewpoints. For example, given a word on the identification test, the McClelland and Rumelhart (1981) formulation suggests that the nodes for enemies receive inhibitory inputs from beginning letters that are not shared with the target. Such input reduces their influence, and the consequences are reduced set size effects. Given an ending on the identification test, the nodes for enemies receive no inhibitory input, since beginning letters are not present. The consequences should be greater category size effects when only endings are exposed. Of course, as suggested earlier, these effects should be more apparent for new than for recently studied words.

The retrieval viewpoint suggests that both category size and strength effects should be reduced for words because of additional information that can be extracted from initial letter positions. These reductions may occur for one of two reasons. First, information from these positions may be used to restrict the breadth of search through ending categories. Hence, the reductions in category size effects for words, relative to those for endings, could emerge because the identification of targets having larger sets should differentially improve. The presence of partial or complete information on beginning letters might curtail the number of items that a subject examines before making a response.

Second, in contrast to the possibility that beginning letters improve the identification of words having larger sets, they may instead decrease the identification of words having smaller sets. The presence of this information may be used to redefine the nonsemantic category. When such information survives the mask, subjects may initiate the retrieval process using beginning, rather than ending, letter information. Research in cued recall indicates that beginning letters can provide a means for initiating retrieval in a manner similar to that used for ending letters (Nelson \& McEvoy, 1984). To the extent that subjects engage in such a process, the reductions in category size for words should emerge because the identification of targets having smaller sets should deteriorate. When words instead of endings are used as identification stimuli, the relative advantage of targets having smaller sets may be lost. Perceptual identification for these items would be less probable because the set defined by initial letters is apt to be larger, on average, than the one identified by endings letters. For example, given the target HUSK, the set defined by $\mathrm{HU}$ is considerably larger than the one defined by USK.

\section{Method}

Design. The experimental design formed a $2^{5}$ mixed-model factorial. Stimulus type (word vs. ending) was manipulated between subjects, and strength, frequency, category size, and prior study (old vs. new) were varied within subject.

Materials and Procedure. The materials and procedures were similar to those used in Experiment 1. Words were exposed for $40 \mathrm{msec}$ and endings were exposed for $250 \mathrm{msec}$ prior to the mask. Otherwise, these two conditions were treated identically. Endings had blanks in place of the missing letters (e.g., _ USK, _ESS).

Subjects. Sixteen subjects were assigned to each condition of stimulus type, with 8 being assigned to each list. Hence, 32 subjects served in the experiment; they were drawn from the same source as those in the previous two studies.

\section{Results}

The probability of recognizing new words given words as test stimuli was . 30 , and that given endings was .31 . Hence, the confounding of exposure duration successfully equated the effectiveness of the two types of stimuli. 
Examination of the principal results shown in Table 3 shows that both category size and strength influenced the likelihood of identification. As expected, this influence was greater for endings than for words. The analysis of variance indicated that category size $[\mathrm{F}(1,30)=54.93$, $\mathrm{MSe}=.034]$ and strength $[\mathrm{F}(1,30)=31.12, \mathrm{MSe}=$ $.040]$ were significant sources, as were the interactions of stimulus type $\times$ category size $[\mathrm{F}(1,30)=15.88, \mathrm{LSD}$ $=.05]$ and stimulus type $\times$ strength $[F(1,30)=27.16$, LSD $=.05]$. When words were shown on the identification test, the probabilities of identifying targets having small and large sets were, respectively, .44 and .38 . When endings were shown, the respective values for small and large sets were .54 and .36 . The LSD shows that set size had reliable effects for each stimulus type, but the interaction shows that these effects were greater for endings. Interestingly, the relative advantage for targets having smaller sets was substantially reduced when words were presented and information on beginning letters was likely to be available. Hence, the category size effect is reduced when words instead of endings are tested, and this reduction is particularly noticeable for targets having smaller sets.

The interaction between stimulus type and strength showed a somewhat similar pattern. When words were shown at test, the probabilities of identifying targets having strong and weak endings were, respectively, .42 and .40 . This difference was not reliable. When endings were shown, the respective values for strong and weak were reliably different -.55 and .35 . Endings that are more strongly connected to their targets produce a substantially higher rate of target identification. This beneficial effect appeared to be completely eliminated when information from beginning letters was available.

These interactions replicated the separate findings reported in Experiments 1 and 2 . In addition, the present experiment replicated the study effect, the frequency effect, and their interaction. Old words $[F(1,30)=89.12$, $\mathrm{MSe}=.091]$ and high-frequency words $[\mathrm{F}(1,30)=$ $55.26, \mathrm{MSe}=.030$ ] were more likely to be identified than were new words or low-frequency words. As before, prior study and frequency interacted $[\mathrm{F}(1,30)=15.38$, $\mathrm{MSe}=.035, \mathrm{LSD}=.05]$. This interaction indicated that prior study substantially reduced the effect of printed word frequency. For studied old words, the probabilities of

Table 3

Probability of Correct Identification as a Function of Strength, Frequency, Category Size, Prior Study, and Stimulus Type

\begin{tabular}{|c|c|c|c|c|c|c|c|c|c|}
\hline \multirow{3}{*}{$\begin{array}{c}\text { Stimulus } \\
\text { Type }\end{array}$} & \multirow{3}{*}{$\begin{array}{l}\text { Prior } \\
\text { Study }\end{array}$} & \multicolumn{4}{|c|}{ Strong } & \multicolumn{4}{|c|}{ Weak } \\
\hline & & \multicolumn{2}{|c|}{ High } & \multicolumn{2}{|c|}{ Low } & \multicolumn{2}{|c|}{ High } & \multicolumn{2}{|c|}{ Low } \\
\hline & & Small & Large & Small & Large & Small & Large & Small & 1 Large \\
\hline Word & $\begin{array}{l}\text { Old } \\
\text { New }\end{array}$ & $\begin{array}{l}.55 \\
.44\end{array}$ & $\begin{array}{l}.50 \\
.38\end{array}$ & $\begin{array}{l}.58 \\
.16\end{array}$ & $\begin{array}{l}.48 \\
.23\end{array}$ & $\begin{array}{l}.54 \\
.50\end{array}$ & $\begin{array}{l}.51 \\
.40\end{array}$ & $\begin{array}{l}.54 \\
.20\end{array}$ & $\begin{array}{l}.39 \\
.19\end{array}$ \\
\hline Ending & $\begin{array}{l}\text { Old } \\
\text { New }\end{array}$ & $\begin{array}{l}.83 \\
.57\end{array}$ & $\begin{array}{l}.66 \\
.35\end{array}$ & $\begin{array}{l}.73 \\
.38 \\
\end{array}$ & $\begin{array}{l}.63 \\
.23 \\
\end{array}$ & $\begin{array}{l}.67 \\
.34 \\
\end{array}$ & $\begin{array}{l}.39 \\
.18 \\
\end{array}$ & $\begin{array}{l}.55 \\
.28 \\
\end{array}$ & $\begin{array}{l}.36 \\
.07 \\
\end{array}$ \\
\hline
\end{tabular}

identifying low- and high-frequency targets were, respectively, .53 and .58 ; for new words, these values ere .21 and .39. A three-way interaction with stimulus type indicated that this pattern was more pronounced when test stimuli were words rather than endings $[F(1,30)=5.17$, $\mathrm{LSD}=.07]$. Nevertheless, reliable frequency effects were obtained for new words, even though only the endings of these targets were shown at test. Hence, given that new words were to be identified, high-frequency words were more likely to be correctly reported regardless of whether the test stimulus consisted of the item itself or just its ending.

None of the remaining 22 sources of variance were reliable, including the main effect of stimulus type and any of the interactions between prior study and either category size or strength. Hence, although the effects of frequency were reduced by prior study, the effects of both category size and strength were not.

\section{Discussion}

The results of Experiment 3 show that the magnitude of category size and strength effects varies with the nature of the test cue presented on the perceptual identification test. Both effects are quite substantial when only endings are presented. Category size effects are substantially reduced and strength effects are essentially eliminated when words are presented. These patterns of identification are consistent with expectations derived from either McClelland and Rumelhart's (1981) model or Jacoby's (1983) hypothesis that perceptual identification involves a retrieval process based on episodically encoded information. However, as in Experiment 1, the magnitudes of category size effects are essentially equivalent for new and for studied words. This finding appears to be incompatible with the perceptual model. In fact, the form of the interactions with stimulus type suggests that subjects may have been using information from beginning letters to redefine the category. Ending set size effects are reduced when words are presented at test, and this reduction is particularly apparent for words having smaller ending sets. Some of the advantage of items having smaller ending sets would be expected to be lost if subjects were retrieving or attempting to retrieve information from categories defined by beginning letters. The effects of ending-to-target strength would also be expected to be reduced if subjects define the relevant set of items in terms of beginning letters.

Aside from replicating the study effect and its interaction with frequency, the results of this experiment also indicate that the word frequency effect in perceptual identification is not dependent on showing whole word units during test. Even though only endings were shown, highfrequency words were more likely to be identified than were low-frequency words. This result is consistent with perceptual models that assume that the word frequency effect is produced by a bias in favor of common words that combines with available sensory information to 
produce the response (e.g., Broadbent, 1967; Morton, 1969). This result is also consistent with the retrieval hypothesis.

\section{GENERAL DISCUSSION}

The results of these experiments provide an independent replication of the study effect and its interaction with printed word frequency (Jacoby \& Dallas, 1981). More important, they show that both nonsemantic category size and strength can have clear effects on perceptual recognition under bright-target/patterned-mask conditions. These category size effects are found for high- and lowfrequency words and for endings that are either strongly or weakly related to their targets. They are not diminished by prior study and are not exaggerated by emphasizing the nonsemantic information during the study trial. Finally, category size effects are found when whole word units or just the endings of these units are shown on the identification test. Hence, under a variety of conditions, words having larger nonsemantic sets are more difficult to identify under perceptually difficult conditions. Similarly, the results of these experiments also show that weaker relationships between ending letters and the target can reduce the chances of identification. Effects of relative strength were observed under conditions of masked word display for items that varied in frequency and category size, and these effects also were not diminished by prior study. The only manipulation that severely curtailed the effects of category size and strength was stimulus type. When whole word units instead of endings were presented on the identification test, the influence of category size was attenuated but not eliminated and the influence of strength was essentially eliminated.

The perceptual model proposed by McClelland and Rumelhart (1981) provides a good explanation for the general findings of this experiment, but it appears to fall short in accounting for some of the specifics. On the positive side, its top-down interactive nature explains the study effect, and, with modifications in the logogen concept, it can also explain the interaction between prior study and frequency (Jacoby, 1983). Prior study of a word strengthens its episodic representation, and this strengthening feeds back to the letter level to promote identification. Low-frequency words presumably would have to benefit more from this study experience than would highfrequency words. Furthermore, the model's assumption of letter-level nodes and the associated recruitment of "friends" and "enemies" predicts and explains both category size effects and their interaction with stimulus type. When an unstudied new word is presented on the perceptual test, it weakly activates other words that share letters with it. The greater the number of incorrect words, the worse the identification performance. However, the influence of these incorrect words is minimal, since, with whole words, information from beginning letters serves to inhibit their activation. From these assumptions, it is possible to predict and explain the weaker category size effects with whole words than with their endings. End- ings omit the initial portion of the word and therefore remove inhibitory effects. Enemies are now as likely to be activated as the target word itself, and the more enemies there are, the worse the performance.

The interaction model clearly handles a major portion of the findings of this series of experiments. However, on the negative side, this model suggests that prior study of the target should both reduce and enhance category size effects, depending on the context of that study. When words are studied in the absence of context, they are strengthened at the word level, and this strengthening should enhance their subsequent perceptibility and reduce the influence of their enemies. In contrast, when words are studied in the presence of contexts that emphasize the nonsemantic features shared by enemies, the influence of these incorrect items should be maximized so that category size effects should be most evident. Unfortunately, the results were inconsistent with either of these potential outcomes. Category size effects were equally apparent, regardless of prior study or emphasis during study. In addition, this model suggests that the weaker category size effects with words than with endings should occur because identification of items having larger sets differentially improves. However, the reduction appeared to result because identification of words having smaller sets tended to deteriorate.

Finally, the interaction model seems to have difficulty in explaining the interaction between strength and stimulus type. The substantial effects of ending strength obtained when endings were shown on the identification test might be explained with the assumption that strongly related targets, like high-frequency words, have lower thresholds. That is, strongly related words might be assumed to be activated more readily and therefore would be more available than weakly related words. This interpretation of the interactive model is consistent with its assumptions and with results obtained with endings as stimuli. However, it is hard to see how this interpretation would explain the absence of strength effects when whole word units are shown. Words that are strongly related to their ending letters should still have a relative advantage, just as high-frequency words have an advantage regaradless of whether the stimulus is a whole word unit or only its ending.

Both the general and the specific findings of the present experiments seem to be more completely explained with the assumption that performance in perceptual recognition and cued recall depends on a common retrieval process (e.g., Jacoby, 1983). In each task, performance is at least partially dependent on the extraction of nonsemantic features from the presented stimulus. This information is retrieved from features-letters that survive the mask or from a stimulus that is physically present. Whatever is extracted can be combined with information preserved from prior study or from prior experience to determine the response.

This retrieval view explains the study effect and its interaction with frequency (Jacoby, 1983; Jacoby \& Dallas, 1981). It also suggests that variations in nonsemantic 
characteristics are likely to have parallel results in the two tasks. When parallel results are not found, differences must be clearly attributable to differences in the nature of the retrieval cues provided or in the nature of the information that can be extracted from them. In the present experiments, the effects of nonsemantic category size and ending strength paralleled results typically obtained in cued recall with rhyme and ending cues. For example, performance is an inverse function of category size in both tasks, and, in each task, it is uninfluenced by emphasizing nonsemantic information at study. As shown in Experiment 2, these parallels are found even when overall levels of identification are so low that subjects are recognizing an average of only $24 \%$ of the items. However, as shown in Experiment 3, the effects of these variables are considerably reduced when whole words, instead of only endings, are presented. The retrieval view suggests that this reduction should be attributed to additional information gained from beginning letters. This information might have been used in one of two ways. For example, partial information on initial letters might have been combined with more complete information on endings to reduce the extent of search within ending categories. Information from initial letters might have been used to help specify the target within the pool of potential items so that the effects of large category size would be particularly weaker for whole words than for endings. The results, however, are not consistent with this alternative.

Another way to use information from beginning letters would be to define the nonsemantic category from initial, rather than from terminal, letter information (e.g., HU__ rather than _USK). To the extent that subjects engage this definition, the influence of both category size defined by the word endings and their relative strength should be reduced. The ending of the word is not being used to identify the relevant search set (e.g., Nelson et al., 1982). Furthermore, these reductions should come about because items having smaller ending categories lose their relative advantage. To the extent that the relevant set is defined from the beginning of the word, little or no advantage should accrue to targets sharing letters with only a few other words. The results are consistent with this alternative and illustrate how the retrieval approach can explain the particular forms of the interactions with stimulus type. Essentially, this view suggests that information gained from initial letters can have a negative effect on identification because it changes and redirects the retrieval process.

The results of the present series indicate that, under conditions of free report, retrieval processes seem to represent an important component of perceptual identification performance under bright-target/patterned-mask conditions. Differences in the effects of variables in perceptual recognition and nonsemantically cued recall seem to arise more from differences in the retrieval cues provided than from differences in task. Hence, perception and memory may rely on the same memory system or systems to mediate performance. This conclusion is essentially in general agreement with theories asserting that perception is a joint product of information presented in the cue and information coming from prior knowledge (Broadbent, 1967; McClelland \& Rumelhart, 1981; Morton, 1969). The primary contribution of the present work is in presenting evidence suggesting that this interaction is mediated through a retrieval process, not through a response bias or through a momentary lowering of thresholds. This suggestion implies that retrieval theories developed in the context of research on memory problems, especially those concerning the role of nonsemantic information, will have potential applications in the field of perception as well (Jacoby, 1983; Jacoby \& Brooks, 1984; Nelson, 1981).

\section{REFERENCES}

Broadbent, D. E. (1967). Word-frequency effect and response bias. Psychological Review, 74, 1-15.

JACOBY, L. L. (1983). Perceptual enhancement: Persistent effects of an experience. Journal of Experimental Psychology: Learning, Memory, and Cognition, 9, 21-38.

JACOBY, L. L., \& BROOKS, L. R. (1984). Nonanalytic cognition: Memory, perception, and concept learning. In G. H. Bower (Ed.), The psychology of learning and motivation: Advances in research and theory (Vol. 18). New York: Academic Press.

JACOBY, L. L., \& DALLAS, M. (1981). On the relationship between autobiographical memory and perceptual learning. Journal of Experimental Psychology: General, 110, 306-340.

JACOBY, L. L. , \& WITHERSPOON, D. (1982). Remembering without awareness. Canadian Joumal of Psychology, 36, 300-324.

Johnston, J. C. (1978). A test of the sophisticated guessing theory of word perception. Cognitive Psychology, 10, 123-153.

MCClelland, J. L., \& Rumelhart, D. E. (1981). An interactive activation model of context effects in letter perception: Part 1 . An account of basic findings. Psychological Review, 88, 375-407.

MorTon, J. (1969). Interaction of information in word recognition. Psychological Review, 76, 165-178.

NELSON, D. L. (1981). Many are called but few are chosen: The influence of context on the effects of category size. In G. H. Bower (Ed.), The psychology of learning and motivation (Vol. 15). New York: Academic Press.

Nelson, D. L., \& McEvoy, C. L. (1979a). Effects of retention interval and modality on sensory and semantic trace information. Memory \& Cognition, 7, 257-262.

Nelson, D. L., \& McEvoy, C. L. (1979b). Encoding context and set size. Journal of Experimental Psychology: Human Learning and Memory, 5, 292-314.

Nelson, D. A., \& McEvoy, C. L. (1984). Word fragments as retrieval cues: Letter generation or search through nonsemantic memory? American Journal of Psychology, 97, 17-36.

Nelson, D. L., McEvor, C. L., \& Friedrich, M. A. (1982). Extralist cuing and retrieval inhibition. Journal of Experimental Psychology: Learning, Memory, and Cognition, 8, 89-105.

PaAp, K. R., Newsome, S. L., McDonald, J. E., \& Schvaneveldt, R. (1982). An activation-verification model for letter and word recognition: The word superiority effect. Psychological Review, 89, 573-598.

R1:ICHFR, G. M. (1969). Perceptual recognition as a function of meaning fulness of stimulus material. Journal of Experimental Psychology, 81, 274-280.

Thorndike, E. L., \& LoRge, I. (1944). The teacher's wordbook of 30,000 words. New York: Teacher's College Press, Columbia University.

(Manuscript received May 13, 1983; revision accepted for publication June 18,1984 .) 